\title{
Testing traffic flow models
}

\author{
Elmar Brockfeld and Peter Wagner \\ German Aerospace Centre, Institute for Transportation Research, \\ Rutherfordstrasse 2, 12489 Berlin, Germany, URL: http://ivf.dlr.de
}

\begin{abstract}
The huge number of different traffic flow models available calls for an attempt to classify, simplify and assess those models. In principle, this is simple to accomplish, since the principal algorithms for assigning quality measures such as $\chi^{2}$ are well known. However, due to a lack of publicly available data, a commonly agreed upon benchmark could not be established up to now. This contribution shows (i) what can be done with such a data-sets, once they are available and (ii) tell about efforts to change the far-from-optimal situation related to the data.
\end{abstract}

\section{So much models, so little time}

The current state of the art of modelling traffic flow is far from optimal. There is a big market of different models of traffic flow that are thought to describe traffic flow into more or less detail. They provide the playing ground for theoreticians, each of which would like to convice the others, that (s)he has gained the biggest understanding of them all, and that her/his model describes the zoo of phenomena like jams, headway distributions, synchronized flow, and even the stock-market [2].

It is true, that most of the models can be taken as a certain approximation to the features observed in real life, and that the models are in a certain kind qualitatively correct. However, when it comes to more detailed questions it starts to become difficult.

Not surprisingly, the result of the usual reviews of those models states something like "for this and that application, this model may be used", but that's most of the time an excuse for being not exact. This small contribution to computational physics tries to go a very small step ahead, by assigning numbers for one given data-set and a number of different models.

\section{Theory}

Models may be classified into microscopic, mesoscopic and macroscopic, with several subclasses (micro can be subdivided further into ODE, map, and cellular automaton models, respectively). When it comes to comparison with reality, most models are tested at most on the level of the so called fundamental diagram. With fundamental diagram it is simply meant the plot of the flow versus density, where both variables flow $q_{i}$ and density $k_{i}$ can be 
assigned in principle to an individual car. In this case, they are defined as:

$$
\begin{aligned}
q_{i} & =\frac{1}{\tau_{i}}=\frac{\Delta x_{i}}{v_{i}} \\
k_{i} & =\frac{1}{\Delta x_{i}}
\end{aligned}
$$

i.e. the flow is defined either as the inverse of the time headway $\tau_{i}$, or, alternatively, as the quotient between front-bumper-to-front-bumper-distance $\Delta x_{i}$ and the velocity $v_{i}$ of a car. Unfortunately, most counting devices record a temporal average of the velocity $\langle v\rangle_{T}$ and the flow $\langle q\rangle_{T}=\Delta N / T$ only, where $T$ is the averaging interval. In most cases the density can be inferred only by using the questionable relationship $\langle k\rangle_{T}=\langle q\rangle_{T} /\langle v\rangle_{T}$. Any textbook comes up with the much better formula $\langle k\rangle_{T}=\langle q\rangle_{T}\left\langle\frac{1}{v}\right\rangle_{T}$.

This is discussed at some length in order to make clear, that this did not contain any idea about a specific model that may be hidden behind such a plot. Sometimes, the fundamental diagram is refered to as a model $q=q(k)$ that may serve as a fixed point model for an underlying microscopic model. With fixed point it is meant, that a car following a car that drives with constant speed $v=v_{\text {lead }}$, this following car will approach the speed $v_{\text {lead }}$ and keep an optimal distance $g=g\left(v_{\text {lead }}\right)$.

One of the interpretations of the fundamental diagram is shown in Fig. 1, in this case there is an additional regime called synchronized flow, where cars do not pick one particular fixed point that is stable but have infinitely many marginally stable states compare the presentation of Boris Kerner in this volume.

But compare this more or less theoretical fundamental diagram with an empirical one. The scatter in the microscopic data is so big, that there didn't seem any particular structure at all. Big question: can models applied successfully to those data?

\section{Testing dynamical models}

Quite in general, any dynamical system can be written as:

$$
\dot{q}(t)=f(q(t), t ; p)+\sum_{j} g_{i j}(q, t) \Gamma_{j}(t) \quad i=1, \ldots, n
$$

where the structure of the noise is given by

$$
\left\langle\Gamma_{j}(t)\right\rangle=0,\left\langle\Gamma_{j}(t) \cdot \Gamma_{k}(t+\tau)\right\rangle=2 \delta_{j k} f(\tau) .
$$

with $f(\tau)$ the noise correlation function, if $f(\tau)=\delta(\tau)$ it is a white noise process, and a coloured noise process otherwise. Here, the phase-space variable $q$ is given by $q=\left\{\left(x_{i}, v_{i}, \ldots\right)\right\}_{i=1, \ldots, N}$, and the variable $p$ denotes a set of parameters. (Of course, in case of a time-discrete dynamics anything can 


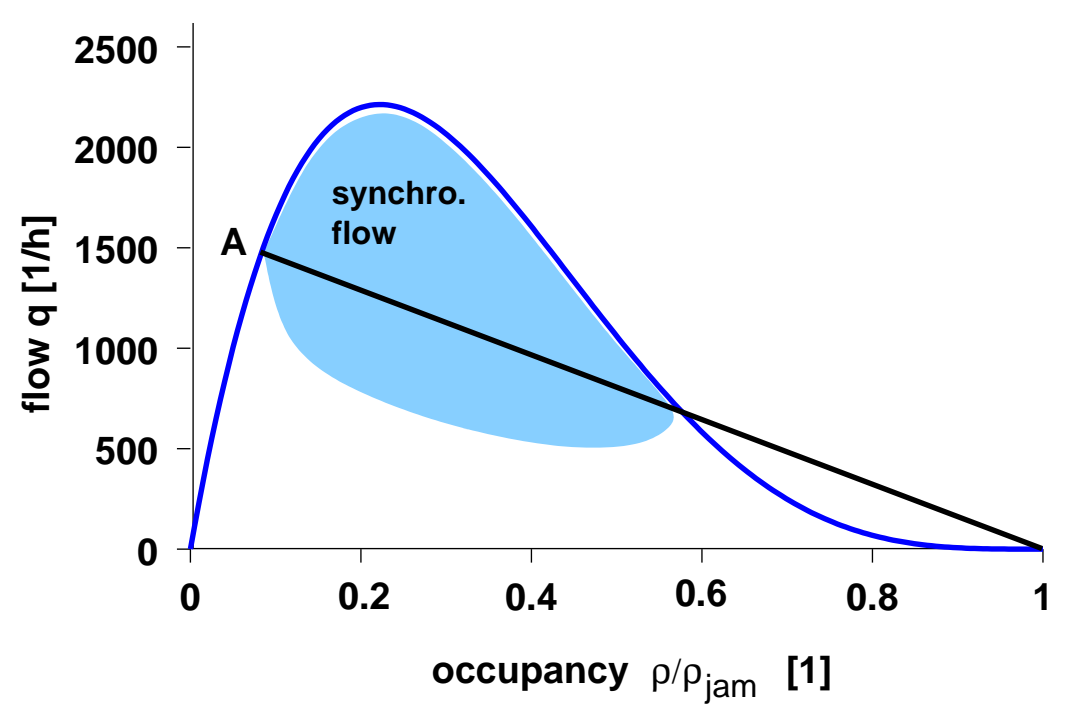

Fig. 1. Theoretical fundamental diagram. Show is the line of virtual homogeneous states, and the illusive state of synchronized flow.

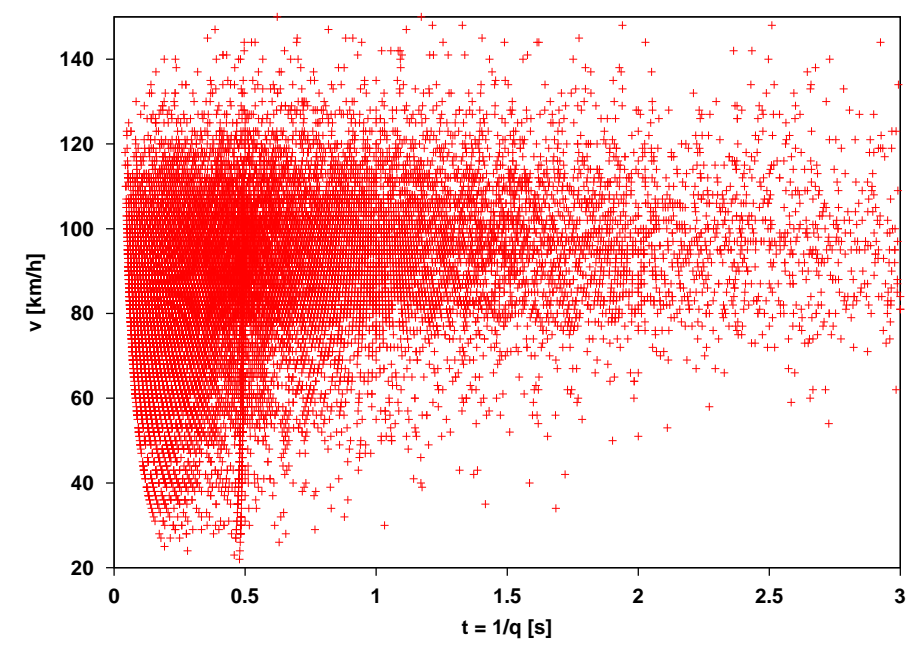

Fig. 2. Empirical fundamental diagram. It looks unfamiliar, since in this case the speed is plotted versus the time-headway, which is the inverse of the flow and can be understood as a scaled distance (distance divided by speed, see 1). The advantage: plotted here are only measured values, not computed ones like the density. 
be carried over by just replacing $\dot{q}$ by the finite difference $(q(t+h)-q(t) / h)$.) In principle, any car $i$ may have its own set of parameters, which is true but would lead to a nightmare of parameters to determine. But note, that this is not just an excuse: certain parameters like the desired acceleration or the maximum speed are definitely different from driver to driver, and even for the same driver from time to time. However, having such a description at hand is physicist's dream, usually, only a small part of a system can actually be observed:

$$
Q(t)=G(q(t))+\Gamma_{o}(t) .
$$

Not surprisingly, in many cases, knowing $Q$ is not enough to infer either the system's state $q$ or it's dynamics $f, g$. This is especially true for traffic, and obtaining really good ideas about $f$ and $g$ for traffic flow constitutes an interesting research program in its own right.

Nevertheless, some work can still be done if a comparatively bad model is at hand, or even none at all, and this is what will be reported next. If there is a model at hand, two things can be done:

- use a Kalman filter to obtain $q$ and the set of free parameters $p$, or, alternatively,

- fit the parameters $p$ directly to the data, by some more or less sophisticated method.

In principle, it can be tried to infer the model directly from the data, an approach whose first results are reported in [1]. This is a different and very interesting avenue of research, however this contribution will stay with the simplest thing to be done, fitting the parameters directly. (The Kalman approach is a fairly complex one if it comes to testing microscopic models, which are the main focus of this contribution.)

Suppose that a certain data-set should be reproduced by a given model. That means that an appropriate error measure, for instance:

$$
e=\frac{\left\langle\left|q_{\mathrm{emp}}-q_{\mathrm{sim}}\right|\right\rangle}{\left\langle q_{\mathrm{emp}}\right\rangle}
$$

should be minimized by searching for a set of parameters that minimizes $e$. (In this equation, $q_{\mathrm{emp}}$ are the observed values and $q_{\mathrm{sim}}$ are the simulated values. This measure gives an easy to understand error measure, the relative absolute error.) The fitting itself can be done by starting a simulation with a given set of parameters, obtaining $e$ and looking then for a new set of parameters $p^{\prime}$ by means of one of the following algorithms:

- guessing (that is really done, and sometimes not as bad as it sounds),

- Nelder-Mead type algorithm (amoeba in Numerical Recipes),

- simulated annealing techniques,

- MJD Powell's COBYLA (implemented in IBM's NAO), 
or any other means of doing a non-linear optimization when derivatives are not available. Of course, the business of doing such an optimization is an interesting and still fertile field of research in its own right, which would deserve another presentation as well. For a short idea, please visit e.g. [13].

\section{The trouble with the data}

Let's go! (i) Getting some data, using one of the algorithms above, this contribution will stick to Nelder Mead, and report the results.

However, it turns out that it is not that simple to obtain meaningful datasets, and even if one such data-set is at hand, you are not allowed to share it with others.

In order to remedy this situation, we are going to record them by our own devices and publish them on our homepage ivf.dlr.de/clearing. Successively we will add (stay tuned!):

- single car data from various locations,

- data from car-following experiments,

- video-based data, and their extracted traffic data,

- probably some aerial data,

- FCD-Data, recorded in Berlin (NDA required!).

together with some test we are going to perform with those data and a number of models whose description is publicly available. Do you have a good idea what to measure? Please tell! And: please tell what you have done with the data. Watch out there for other goodies as well, e.g. the open source traffic simulation project SUMO (www.sourceforge.org/sumo).

\section{Daganzo's data}

Here is an example, and what to do with it. Some years ago, Carlos Daganzo recorded a nice data-set [4] which will be used in the following to perform some model testing. Along a one-lane road where overtaking was not possible, eight observers were positioned, each of them equipped with a lap-top to record the times a certain car passes him/her. One lead car that drove several times along this road provided the initial car number, therefore traffic flow is recorded by so called $N(t)$-curves where $N$ is the number of cars that passed a certain position up to a certain time $t$. Typical recording errors are of the order of 5 cars out of 600 by which the counts of the different observers differed. In the following a data-set has been used that has been corrected for those small mistakes, we will make it available on our homepage as well. The time accuracy can be assumed as of the order of the one second resolution that is reported in Daganzo's data. The Fig. 3 shows a sketch of the recoding site, in the following some additional informations are given: 


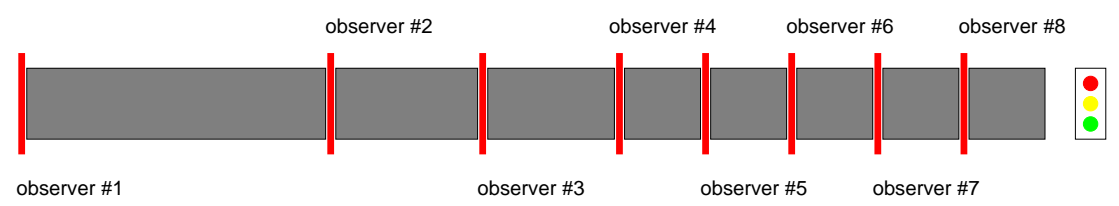

Fig. 3. Sketch of the Pablo Dam Road where the data used in this article have been recorded.

- The total length of the one-lane road is about $6.4 \mathrm{~km}$,

- there is a traffic light at the end, controlling the flow into another highway,

- speed-limit of about $25 \mathrm{~m} / \mathrm{s}$ is imposed on this road,

- two days of data have been recorded, each of them from 7 to $9 \mathrm{am}$.

The traffic light at the end causes a congestion, when demand rose during the morning rush-hour. This can be seen most easily in the Fig. 4 where the travel times on segment one and the last segment six are plotted. Only six segments have been used, since the data from oberserver \# 5 are partly missing, so they were discarded alltogether in order to simplify the analysis.
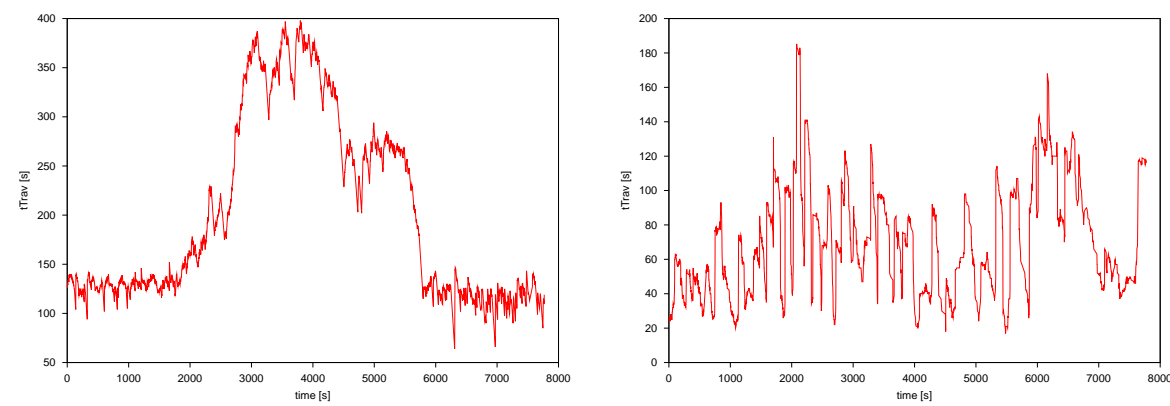

Fig. 4. Travel time as function of the number of seconds that have passed since starting of the record. It can be seen very clearly, how the small jams created by the traffic light travel backwards. Note however, that the merge more or less into one big jam wave that starts to flatten out at the first observer.

\section{The Contest}

\subsection{Building a reference model}

How well can models describe this situation? In order to have some model for comparison, a very simple reference model will be constructed. Since the data are from Carlos Daganzo, a variant of his cell transmission model will 
be used [5]. This is basically a very simple queueing model, which takes care of continuity \& capacity constraints. The model is specified further by:

- The road is divided into small cells of length 13 cars $(100 \mathrm{~m})$, where

- any cell has a maximum occupancy $\left(N_{i}\right)$, an actually occupancy $n_{i}$ and a maximum flow out $c_{\max }$.

- Cars can pass to the next cell only if there is enough room, and the

- number of cars that pass from one cell $i$ to the next one $i+1$ during one time-step are computed as $\Delta N(i \rightarrow i+1)=\min \left\{v n, c_{\max }, w\left(N_{i+1}-n_{i+1}\right\}\right.$ the fundamental diagram is implemented as $c(n)=n<n_{c} ? v_{1} n: v_{2}(N-$ $n)$

- The size of a time-step $h$ is just the minimum travel time, i.e. $h=$ $L_{i} / v_{\max }$.

In order to compare this model directly to the data, some more microscopic details have been added. After some trial and error, it converged on the following set of rules:

- upon entering the cell, any car $i$ gets assigned an assumed leaving time $t_{i}^{\text {exit }}=t_{i}^{i n}+t_{\min }$, where $t_{\min }$ is the minimum travel time.

- Each cell is modelled as a short first-in-first-out queue,

- the capacity is implemented by noting that a flow is just an inverse time headway. Therefore, cars can leave only if the car that has left the cell before is at minimum $\tau \approx 1 / C$ away, where $c$ is the maximum possible flow.

- The entry into the next cell can be delayed if space is scarce there. This is being done by adding a penalty time before allowing to leave cell $i$ that is proportional to $n_{i+1}$.

This cell transmission and its microscopic counterpart are well-known for the fact that jams are not stable, they decay with a relatively small timeconstant. In order to implement this feature, the entry into the next cell has to be delayed a little when compared with the approach above if $n_{i}>n_{+1}$ [15].

In the presentation given at the comphys02-conference, a travel time function has been used that was a function of the number of cars in the cell. Surprisingly, this basically has not effect on the outcome of a simulation of the queue-model, therefore it is not used anymore.

Probably, there is still a better way to translate cell transmission into a microscopic model, see for instance [14].

This simple model has four parameters $\tau_{\min }, q_{\max }, \alpha$ and $\beta$, where $\alpha$ and $\beta$ are just the proportionality constants in the computation of the penalty term, $T_{\text {pen }}=\alpha n_{i+1}$, the same for $\beta$ instead of $\alpha$. 


\subsection{Technicalities}

In order to actually start a simulation, some technical problems have to be solved. These are basically the boundary conditions, which are complicated by the fact that the observers have not recorded the speeds. So, for the inflow, velocities were set to the so called safe velocity of the corresponding microscopic model, which may cause in principle travel times that are too short. The outflow condition was modelled at the position of observer \# 8 by a fictitious traffic light that is switched by the number of cars that have left the road. The light is on red if $n_{\text {out }}^{\mathrm{sim}} \geq n_{\text {out }}^{\mathrm{emp}}$, and it switches to green once this inequality is false. Obviously, this is not the best possible solution, since the models have to deal with the discontinuity of switching the traffic light.

The reference model, it turns out, gives reasonable results. Best values obtained by the Nelder Mead algorithm was about $18 \%$ error for the travel times between the observers, some more details are discussed in the figure caption of Fig. 5.
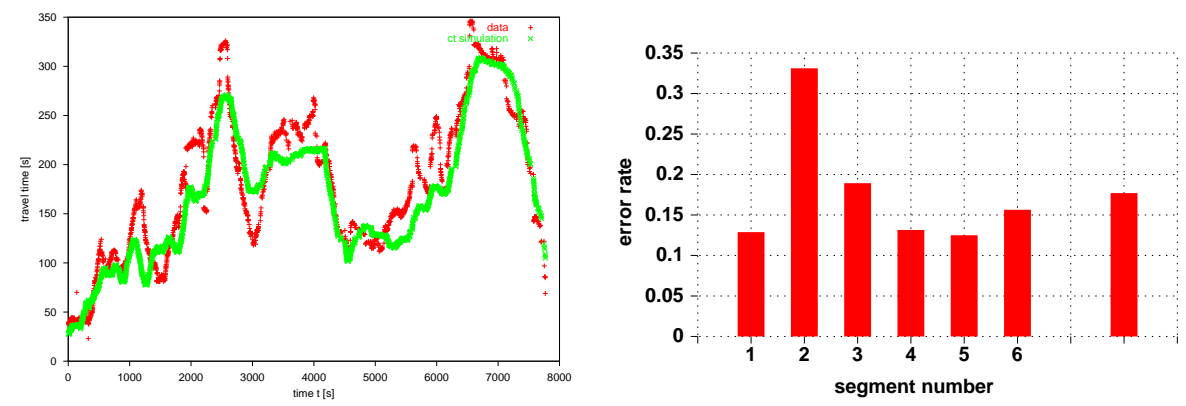

Fig. 5. Left figure: travel time as function of time for the simulation result, compared to the data. It can be seen, that even this simple model fits the data quite well. Right figure: The distribution of the errors for the different observers. It can be seen, that the second segment has the largest error, a pattern that is seen for all of the models.

\subsection{Now the rest of the pack}

Interestingly, no model could do much better. Even the much more sophisticated models did not yield an error rate substantially better than the $17 \%$ of the cell transmission model. Since a winner must be named, we do that in the following, note however, that the differences to the second best model is very small and may subject to change: optimization is sometimes more an art than science, and $1 \%$ decrease in the error rate may be achieved e.g. by making minor changes to the simulation set-up. 


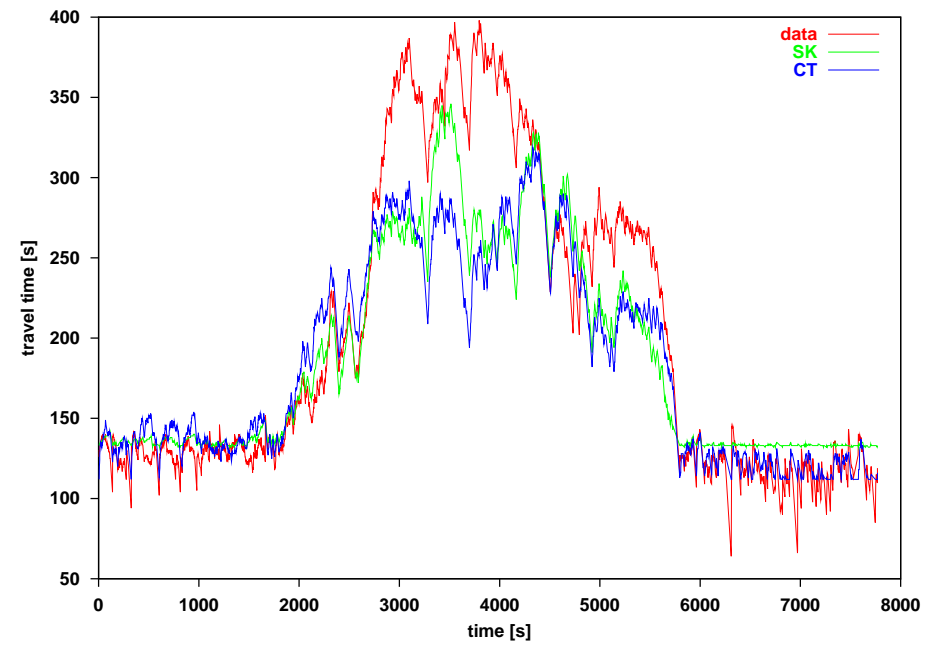

Fig. 6. Travel times for the SK-model below on the first segment, in comparison with the data and the best values obtained by the cell transmission model. Note, that there are very few differences in the performance of the two models.

The model that performed best is, we are really sorry about that, our own. It is a variant of the Gipps-model [12], invented some years ago by Stefan Krauß [9]. The basic idea of the model is still fairly simple: in order to avoid crashes, brake realistically, and try to keep the speed always smaller than a certain maximum safe velocity $v_{\text {safe. }}$. If braking happens to occur with constant deceleration, then $v_{\text {safe }}$ can be computed exactly:

$$
v_{\text {safe }}=-b \tau+\sqrt{b^{2} \tau^{2}+\tilde{v}^{2}+2 b g} .
$$

Note, that this model is, like the CA-model, a kind of time-discrete OVmodel, however with an optimal velocity that additionally depends on the speed of the leading vehicle. It can be understood as the special case of an optimal velocity model differential equation which has been time-discretized with the (too big) time step $h=\tau$ :

$$
\tau \dot{v}=v_{\text {safe }}-v
$$

Unfortunately, even this result came with a drawback: the optimal parameters of the model found need a very large acceleration of around $a \approx 1.8 \mathrm{~m} / \mathrm{s}^{2}$ which are not very realistic, and which are different from the parameters Krauß has found [8].

\subsection{Finally, the Rest of the Pack (Preliminary Results!!!)}

Below, the results of all the models tested so far are listed. It is important to note, that these results are preliminary since nonlinear optimization is always 
more like black magic than like science. The abbreviations used in the plots are translated as follows: IDM = intelligent driver model [11], CA = cellular automaton model [7], ZPR is the Gipps-like model mentioned above, CT is the cell transmission model above, $\mathrm{VDR}=$ velocity dependent randomization, a variant of the CA-model [16], OVM = optimal velocity model [10] (but see also [15] for the reference to a similar model). The recent model of Kerner [3] could not have been implemented bugfree uptonow, the same is true for the new CA-model with braking lights [6].

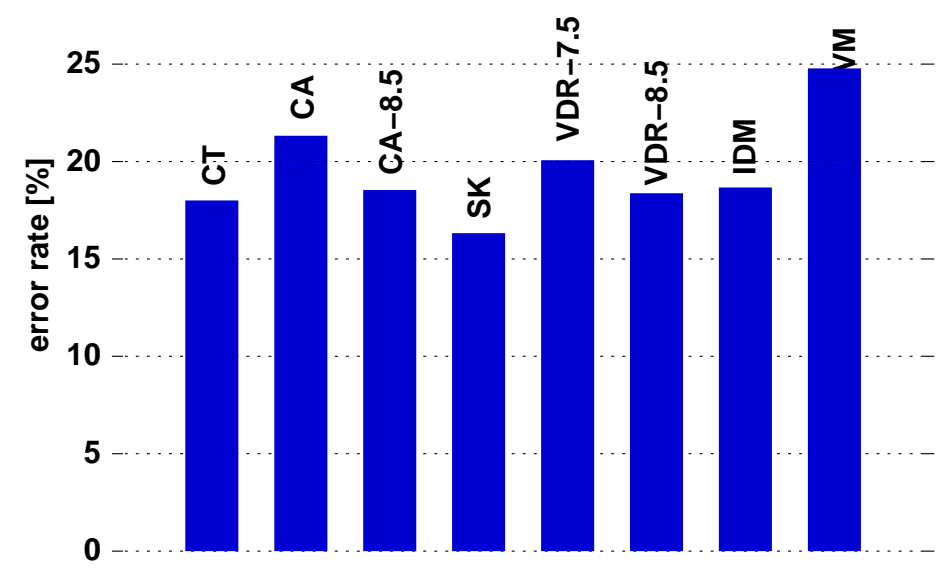

Fig. 7. Comparison of the different models.

\section{Conclusions \& further plans}

Physicists can measure certain numbers with an accuracy better than $10^{-12}$, but in this case the models just display, that either there are no good models actually available, or that there is an unexplainable rest of about $20 \%$ in the behaviour of the drivers that is completely random, or far beyond our current description. It remains to be seen whether this is true for other datasets and other models as well. Another point is that we have to utilize the second day of data as well just to discuss how well a given model is capable of generalizing.

In order to improve the models, a number of suggestions will close this contribution:

- add some psycho facts, e.g. about recognition, learning, comfort, planning,

- people are sometimes planning their behaviour, 
- acceleration usually adopts on time-scales of $1 \ldots 2$ second $\Rightarrow$ use $\dot{a}^{-}$ models?,

- people drive sloppy, a phenomena that is hard to model.

\section{Acknowledgements}

We would like to thank Carlos Daganzo for putting this beautiful data-set on his web-site. Additionally, we would like to thank Chris Cassir, Georg Hertkorn, Boris Kerner, Kai Nagel, Michael Schreckenberg and Richard Woesler for discussions, and the Federal German Ministry of Education and Research for financial support.

\section{References}

1. Kriso, S., Friedrich, R., Peinke J., Wagner P., Reconstruction of dynamic equations for traffic flow, submitted to Physica A (2002).

2. Helbing, D., Batic, D., Schoenhof, M., and Treiber, M., Modelling Widely Scattered States in 'Synchronized' Traffic Flow and Possible Relevance for Stock Market Dynamics, http://de.arxiv.org/abs/cond-mat/0108548 (2001).

3. Kerner, B.S. and Osipov, X., Cluster Effect in Initially Homogeneous Traffic Flow, 35 L31 - L43 (2002)

4. The data of Carlos Daganzo are publicly available on his web-site www.ce.berkeley.edu/ ${ }^{\sim}$ daganzo.html

5. Daganzo, C. F., The cell transmission model: a simple dynamical representation of highway traffic, Transp. Res. B 28269 (1994).

6. Knospe, W., Santen, L., Schadschneider, A., and Schreckenberg, M., Towards a realistic microscopic description of highway traffic, J. Phys. A33, L477 (2000).

7. Nagel, K., and Schreckenberg, M., J. Physique I 22221 (1992).

8. Krauß, S., Microscopic modelling of traffic flow: Investigation of Collision Free Vehicle Dynamics, University of Cologne, 1998.

9. Krauß, S., Wagner, P., and Gawron, C., Metastable states in a microscopic model of traffic flow, Phys. Rev. E, 555597 - 5605 (1997).

10. Bando, M, Hasebe, K., Nakayama, A., Shibata, A., and Sugiyama, Y., Dynamical model of traffic congestion and numerical simulation, Phys. Rev. E, 511035 - 1042 (1995).

11. Treiber, M., Hennecke, A., Helbing D., Derivation, Properties, and Simulation of a Gas-Kinetic-Based, Non-Local Traffic Model, Physical Review E, 59239 253 (1999).

12. Gipps, P. G., A behavioural car following model for computer simulation, Transp. Res. B 15105 - 111 (1981).

13. Surf to http://plato.la.asu.edu/guide.html.

14. Heidemann, D., A Queueing theory model of nonstationary traffic flow, Transportation Science 35, $405-412$ (2001).

15. Newell, G. F., Theories of instability in dense highway traffic, J. Oper. Res. Soc. Japan, 5, 9 - 54 (1962).

16. Barlovic, R., Santen, L., Schadschneider, A., and Schreckenberg, M., Metastable States in Cellular Automata for Traffic Flow, Europ. Journ. Phys. B 5, 793 (1998) 